\title{
The vorticity dynamics of instability and turbulence in a breaking internal gravity wave
}

\author{
David C. Fritts ${ }^{1}$, Steve Arendt ${ }^{1}$, and Øyvind Andreassen ${ }^{2}$ \\ ${ }^{1}$ Colorado Research Associates/NWRA, 3380 Mitchell Lane, Boulder, CO 80301, U.S.A. \\ ${ }^{2}$ Norwegian Defence Research Establishment, P.O. Box 25, N-2007 Kjeller, Norway
}

(Received July 24, 1998; Revised December 20, 1998; Accepted December 20, 1998)

\begin{abstract}
We perform a three-dimensional simulation of a breaking internal gravity wave in a stratified, compressible, and sheared fluid to investigate the vorticity dynamics accompanying the transition from laminar to turbulent flow. Baroclinic sources contribute preferentially to eddy vorticity generation during the initial convective instability of the wave field, yielding counter-rotating vortices aligned with the external shear flow. These vortices enhance the spanwise vorticity of the shear flow via stretching and distort the spanwise vorticity via advective tilting. The resulting vortex sheets undergo a dynamical (Kelvin-Helmholtz) instability which rolls the vortex sheets into tubes which link, in turn, with the original streamwise convective rolls to produce a collection of intertwined vortex loops. Following the formation of discrete vortex loops, the most important interactions are the self-interactions of single vortex tubes and the mutual interactions of adjacent vortex tubes in close proximity. The initial formation of vortex tubes from the roll-up of localized vortex sheets imposes axial vorticity variations having both axisymmetric and azimuthal wavenumber two components. Axisymmetric variations excite axisymmetric twist waves, or Kelvin vortex waves, which propagate along the tubes, drive axial flows, and deplete and fragment the tubes. Azimuthal wavenumber two variations excite $m=2$ twist waves on the vortex tubes which amplify and unravel single vortex tubes into pairs of intertwined helical tubes. Other interactions, judged less fundamental to the turbulence cascade, include reconnection among tube fragments, mutual stretching of orthogonal tubes in close proximity, excitation of azimuthal wavenumber one twist waves, and the continual roll-up of weaker vortex sheets throughout the evolution. Collectively, these vortex interactions result in a rapid cascade of energy and enstrophy toward smaller scales of motion.
\end{abstract}

\section{Introduction}

Transitions from laminar to turbulent flows and the dynamics of the turbulence cascade are longstanding problems relevant to many fields of fluid dynamics. Until recently, progress was confined to studies of the stability of various flows to small perturbations, to observations of instability and turbulence dynamics in the atmosphere and oceans, or to laboratory analogs of these flows. However, the continuing evolution of computational capabilities has now made possible fluid dynamics simulations at sufficiently high Reynolds numbers to describe instability and turbulence processes occurring in geophysical flows.

In the stably stratified atmosphere and oceans away from boundaries, turbulence is believed to arise largely due to wave-induced or shear-flow instabilities. Stability studies and linear wave structure suggest that internal gravity waves contribute to turbulence generation via both convective and dynamical instabilities, with convective instabilities predominant at high intrinsic frequencies and shear instabilities likely more prevalent nearer inertial frequencies (Hodges, 1967; Fritts, 1984; Dunkerton, 1984; Fritts and Rastogi, 1985; Fritts and Yuan, 1989; Winters and Riley, 1992;

Copy right(C) The Society of Geomagnetism and Earth, Planetary and Space Sciences (SGEPSS); The Seismological Society of Japan; The Volcanological Society of Japan; The Geodetic Society of Japan; The Japanese Society for Planetary Sciences.
Dunkerton, 1997; Sonmor and Klaassen, 1997). These inferences are supported by more recent three-dimensional (3D) modeling of wave breaking in atmospheric and oceanic contexts (Andreassen et al., 1994a; Fritts et al., 1994; Isler et al., 1994; Winters and D’Asaro, 1994). These studies found the dominant instability to be a shear-aligned, counter-rotating convective instability with both buoyant and shear sources of eddy kinetic energy. Subsequent studies by Fritts et al. (1996a, 1997) found the same instability character to persist in the presence of transverse mean shears and for waves at lower intrinsic frequencies, respectively.

Shear, most commonly Kelvin-Helmholtz (or KH), instabilities may arise due to unstable mean or wave-induced shears (Fritts and Yuan, 1989; Yuan and Fritts, 1989; Dunkerton, 1997; LeLong and Dunkerton, 1998a,b). For sufficiently inviscid flows, these motions undergo a transition to 3D structure similar to that found in breaking gravity waves, with more recent numerical simulations again consistent with the predictions of linear stability theory (Klaassen and Peltier, 1985; Palmer et al., 1994, 1996; Caulfield and Peltier, 1994; Scinocca, 1995; Fritts et al., 1996b; see also Hill et al., 1999, in this issue). Thus, the initial transitions from laminar, quasi-two-dimensional (2D) to 3D flows are now reasonably understood in sheared and stratified fluids. What has received less attention to date are the further transitions to and dynamics within fully turbulent flows. 
Significant progress has occurred, nevertheless, in describing the statistical properties and the character of vortex structures in numerical and observational studies of homogeneous turbulence in sheared and unsheared geophysical fluids (Rogers and Moin, 1987; She et al., 1990; Vincent and Meneguzzi, 1991, 1994; Sandham and Kleiser, 1992; Cadot et al., 1995; Metais et al., 1995). Other studies have employed forcing at large scales or initial equilibrium distributions of variance in one or more fields to examine evolutionary flow characteristics at smaller scales of motion (Herring and Kerr, 1993; Erlebacher and Sarkar, 1993; Jimenez et al., 1993; Vincent and Meneguzzi, 1994; Gerz et al., 1994; and references therein). The consequence of these dynamics is often found to be a series of "horseshoe" or "hairpin" vortices in boundary layer or sheared flows (Robinson, 1991; Gerz, 1991; Sandham and Kleiser, 1992; Metais et al., 1995).

The emerging vortices exhibit a range of interactions and dynamics which are not well understood at present. For example, oscillations of vortex tubes were studied in the laboratory (Hopfinger et al., 1982; Maxworthy et al., 1985) and in numerical simulations (Melander and Hussain, 1994, 1995; Schoppa et al., 1995), whereas the unraveling and fragmentation of vortex tubes and vortex reconnection events were noted by other authors (Pumir and Siggia, 1990; Boratav et al., 1992; Shelley et al., 1993; Cadot et al., 1995). Based on such observations, several authors sought a theoretical understanding of vortex oscillations (Melander and Hussain, 1994, 1995; Schoppa et al., 1995; Arendt et al., 1997).

Our purposes in this paper are to describe both the vorticity dynamics accompanying the transition from laminar to turbulent flow and the subsequent vortex interactions which appear to drive the turbulent cascade of energy and enstrophy to smaller scales of motion. We adopt this vorticity dynamics perspective, as opposed to our previous studies which examined instability energetics, as this is the approach which provides the clearest understanding of the dynamical interactions among adjacent vortices. To describe the vorticity evolution over a wider range of scales and to provide enhanced definition of the small-scale structures, we also increased the model resolution and reduced dissipation relative to our previous studies. The resulting vorticity evolution is seen to comprise three stages. The first is the primary convective instability described previously by Fritts et al. (1994, 1996a). The resulting convective rolls stretch the vorticity of the background shear flow, and so create intense localized vortex sheets. This leads to the second stage of the evolution: the dynamical (generalized KH) instability of these spanwise vortex sheets ${ }^{1}$. The spanwise vortex sheets roll up into tubes which link, through tilting, twisting, and stretching, with the initial shear-aligned (streamwise) counter-rotating convective vortices to form a collection of intertwined vortex loops. The third stage of the evolution involves increasingly rapid and complex interactions among the vortex loops which drive the vorticity field toward a more isotropic state at small scales.

The stages noted in our study are similar to those described by Vincent and Meneguzzi (1994) in the evolution of homo-

\footnotetext{
${ }^{1}$ In our discussion, the horizontal directions $x$ and $y$ along and normal to the direction of wave propagation will be referred to as streamwise and spanwise, respectively.
}

geneous turbulence, with vortex sheet formation preceding roll-up via dynamical instability and vortex interactions driving the evolution to smaller scales of motion. Our results thus provide partial verification of earlier predictions of the features of such an evolution by Betchov (1957) and Lundgren (1982), such as the intensification of vorticity sheets preceding the formation of vortex tubes. The vortex loops that we find in our simulations are also very similar to those found in other turbulent flows (e.g., Robinson, 1991; Sandham and Kleiser, 1992; Gerz et al., 1994; Metais et al., 1995). Thus, the interactions of the loops we observe are likely to be representative of such turbulent transitions in many flows.

Our paper is organized as follows. The mathematical formulation is described in Section 2. An overview of the enstrophy and vorticity evolutions is provided in Section 3. Section 4 identifies the dominant vortex interactions which drive the cascade to smaller scales of motion. These include vortex sheet intensification and roll-up, orthogonal vortex stretching, and mode-zero and two twist waves on the vortex tubes. Other interactions identified, but apparently less significant in the cascade, include mode-one twist waves, pairing events among aligned tubes, and viscous reconnection of vortex tubes or fragments in close proximity. These interactions are described in Section 5. The evolutions of the enstrophy spectra are displayed in Section 6, and our conclusions and a discussion of the relevance of these results to general sheared turbulence studies are provided in Section 7.

\section{Mathematical Formulation \\ 2.1 Model description}

Breaking and instability of an internal gravity wave is simulated using a nonlinear, compressible spectral collocation code described in detail by Andreassen et al. (1994a) and Fritts et al. (1996a) for studies of wave breaking and instability structures in parallel and skew shear flows. It solves the equations describing nonlinear dynamics in a compressible, stratified, and sheared fluid using a spectral representation of viscous and diffusive effects. These equations are written as

$$
\begin{gathered}
\frac{\partial \rho}{\partial t}+\nabla \cdot(\rho \mathbf{v})=0, \\
\rho \frac{d \mathbf{v}}{d t}=-\nabla p+\rho \mathbf{g}+\mathbf{F}+\mathbf{P}, \\
\frac{d p}{d t}+\gamma p \nabla \cdot \mathbf{v}=Q,
\end{gathered}
$$

where $\mathbf{v}=(u, v, w)$ is velocity, $\rho$ and $p$ are density and pressure, $\mathbf{g}$ is the gravitational acceleration, and $\gamma$ is the ratio of specific heats. The density and pressure are related to temperature through the equation of state, $p=\rho R T$, and the potential temperature, defined as $\theta=T\left(p_{0} / p\right)^{R / c_{p}}$, is used as an approximate tracer of fluid motions.

For convenience, all variables are nondimensionalized using the density scale height $H=(d \ln \rho / d z)^{-1}$, sound speed $c_{\mathrm{s}}$, with $c_{\mathrm{s}}^{2}=\gamma g H$, a time scale $H / c_{\mathrm{s}}$, and reference temperature $T_{0}$, density $\rho_{0}$, and pressure $p_{0}$. We also assume the atmosphere to be initially isothermal, yielding a nondimensional buoyancy frequency squared $N^{2}=(\gamma-1) / \gamma^{2}$ and a corresponding nondimensional buoyancy period $T_{\mathrm{b}} \simeq 14$.

The additional terms on the right sides of the momentum and energy equations include a body force $\mathbf{F}$ to excite 
the primary gravity wave and spectral representations of the diffusion terms, $\mathbf{P}$ and $Q$, to describe the effects of viscosity and thermal diffusivity. The forms of these diffusion terms are described in detail by Andreassen et al. (1994a,b). Here, it is important to note only that these terms represent second-order dissipation at large wavenumbers, but have no influence on wave and instability structures at larger scales of motion. This form of dissipation provides an accurate description of energy removal within the motion spectrum at high wavenumbers and reduces the spectral scattering of energy to larger scales often accompanying higher-order dissipation schemes (Jimenez, 1994). The diffusion terms $\mathbf{P}$ and $Q$ were chosen to yield a normalized kinematic viscosity of $v \simeq 0.015$ and a Prandtl number $\operatorname{Pr}=v / \kappa=0.7$ at the level of wave breaking.

Equations (1) are solved in Cartesian coordinates, $(x, y, z)$, using the spectral collocation method described by Canuto et al. (1988). A Fourier/Chebyshev representation of the solution using trigonometric functions and Chebyshev polynomials is employed to describe the horizontal and vertical structures, respectively. Additionally, a grid-stretching procedure is employed to achieve a more uniform distribution of grid cells in the vertical (see Andreassen et al., 1994a, and Fritts et al., 1996a, for additional details).

As in our previous studies, our simulation is performed in a physical domain composed of two model domains to make efficient use of computer resources and to provide high spatial resolution only where needed to describe the evolution of instability and smaller-scale structures. Wave excitation is performed in a low-resolution lower domain, with wave breaking and instability confined to a higher-resolution upper domain. Nondimensional domain sizes are specified to be $\left(x_{10}, y_{10}, z_{10}\right)=(4,2,4)$ and $\left(x_{20}, y_{20}, z_{20}\right)=(4,2,1.5)$ for the lower and upper domains, respectively, with $z=0$ defined at the lower boundary of the lower domain. Finally, we used $\left(N_{x}, N_{y}, N_{z}\right)=(192,96,129)$ collocation points in the upper domain to provide approximately isotropic resolution of small-scale structures arising due to wave breaking and instability and to insure accurate descriptions of the various sources and sinks of small-scale vorticity.

Boundary and forcing conditions are likewise as described earlier by Andreassen et al. (1994a) and Fritts et al. (1996a). Solutions are assumed to be horizontally periodic, while characteristics of the nonlinear equations are used both to insure continuity of the field variables and their derivatives at the interface between domains and to impose open boundary conditions at the upper boundary of the upper domain and the lower boundary of the lower domain. A gravity wave is excited in the lower domain via a vertical body force having a Gaussian distribution in the vertical, a wavelength equal to the domain dimension in $x$, and an amplitude that ramps up, remains uniform, then ramps down over a time span of $t=60$. The wave frequency is specified so as to lead to a critical level for the wave in the upper domain where the mean streamwise motion achieves an amplitude of $U_{0}(z)=0.2$. The mean flow is described by a cosine and increases from $U_{0}=0$ to $U_{0}=0.3$ across the upper domain. These choices result in a gravity wave that achieves convective instability at an intrinsic frequency $\omega_{i} \sim N / 6$ and extending over a depth $z_{2} \simeq 0.15$ (corresponding to a dimensional size of $\sim 1$ $\mathrm{km})$. Additional details on the initial conditions, evolving mean structure, and numerical procedures can be found in Andreassen et al. (1994a) and Fritts et al. (1996a).

\subsection{The vorticity equation}

The equation describing the evolution of the components of vorticity, $\omega_{i}=(\nabla \times \mathbf{v})_{i}$, may be written

$$
\begin{aligned}
& \frac{d \omega_{i}}{d t}=\omega_{j} S_{i j}+\left(\frac{\nabla \rho}{\rho} \times \frac{\nabla p}{\rho}\right)_{i} \\
& -\left[\omega \nabla \cdot \mathbf{v}+\frac{v}{\mathbf{3} \rho} \frac{\nabla \rho}{\rho} \times\left(\mathbf{3} \nabla^{\mathbf{2}} \mathbf{v}+\nabla(\nabla \cdot \mathbf{v})\right)-\frac{v}{\rho} \nabla^{\mathbf{2}} \omega\right]
\end{aligned}
$$

where summation over repeated indices is assumed. Here, $S_{i j}=\frac{1}{2}\left(\partial_{i} v_{j}+\partial_{j} v_{i}\right)$ is the strain tensor. The term $\omega_{j} S_{i j}$ contains the tilting and twisting of the vorticity vector (offdiagonal terms of $S_{i j}$ ) and the stretching of the vorticity vector (diagonal terms of $S_{i j}$ ) by a flow $\mathbf{v}$. The term $[(\nabla \rho / \rho) \times$ $(\nabla p / \rho)]_{i}$ is the baroclinic source/sink of vorticity, which is non-zero if the surfaces of constant pressure and density are not co-aligned; the baroclinic term describes the creation of vorticity by the torque of buoyancy force on the fluid. The strain and baroclinic terms are the most important for understanding the vortex dynamics in the present paper. The term in square brackets on the right side of Eq. (2) includes contributions due to compressibility and the spectral viscosity and thermal diffusivity employed in our formulation. Both the compressibility and the dissipation are of minor importance for the instability structures discussed in this paper because of the large scales and small velocities of the flow.

\section{Definition of a Vortex}

The vorticity in our simulation results is concentrated into two main geometries: sheets and tubes. Sheets can be flat or curved, but have one dimension much smaller than the other two. Tubes are cylinders with roughly circular cross section. These are not to be confused with vorticity fieldlines which follow the vorticity field independent of magnitude.

To define tubes more quantitatively, it is useful to have a more formal definition of a vortex tube. For this, we adopt the mathematical framework introduced by Jeong and Hussain (1995) and employ the tensor defined as

$$
\mathbf{L}=\mathbf{S}^{2}+\Omega^{2}
$$

Here, $S_{i j}=\frac{1}{2}\left(\partial_{i} v_{j}+\partial_{j} v_{i}\right)$ as before, and $\Omega_{i j}=\frac{1}{2}\left(\partial_{i} v_{j}-\right.$ $\left.\partial_{j} v_{i}\right)$ is the rotation tensor. $S_{i j}$ and $\Omega_{i j}$ are the symmetric and antisymmetric components of the velocity gradient tensor $\nabla \mathbf{v}$. As $\mathbf{L}$ is symmetric, it has only real eigenvalues (ordered $\lambda_{1} \geq \lambda_{2} \geq \lambda_{3}$ ). A vortex will be defined as a region where the middle eigenvalue, $\lambda_{2}$, is negative, and less than an appropriate cutoff value. An important point is that, for our flow regime, a vortex defined in this manner is based on the local tendency for flow rotation rather than on vorticity magnitude. As such, this definition provides greater sensitivity to vortex structures that are weak, but coherent, and an ability to identify such structures at early stages of the flow evolution. We have found that this definition also yields consistent vortex identification at later stages when the structures are highly complex. Finally, because $\lambda_{2}$ is based on flow rotation, vortex sheets are not prominently displayed by $\lambda_{2}$ even if their vorticity is large. 


\section{Overview of Enstrophy and Vorticity Evolutions}

In this section, we provide an overview of the evolution of enstrophy and the emergence of coherent vortices within the breaking wave. The evolution of the breaking wave is illustrated by an isosurface of potential temperature at four times in the upper panels of Plate 1, with the wave propagating to the right. This particular isosurface is shown because it resides within the region of vigorous wave breaking. Noting that a buoyancy period is $T_{\mathrm{b}}=14$ in our non-dimensional units, we see that the entire interval displayed spans less than a buoyancy period; this evolution is both more rapid and more vigorous than was observed in our previous lower-resolution, more viscous simulations.

In the first stage of evolution of the breaking wave, regions of convective instability arise (see, e.g., the overturning isosurface at $t=65$ in Plate 1) from both a compression of the vertical wavelength and an increase of the wave amplitude with height, due to a decrease of wave intrinsic frequency in shear and of mean density with upward propagation. This convective instability results in the formation of streamwise counter-rotating vortex pairs which cause a transition from 2D to 3D flow. These vortex pairs are visible in the top two panels of Plate 2, which show volumetric renderings of the $\lambda_{2}$ eigenvalue introduced in the previous section. Plate 2 displays the entire evolution of the vorticity field (viewed from the bottom with streamwise right and spanwise down), from the first stages of convective instability through the transition to and the evolution within the turbulence spectrum. Strong vortices are colored by opaque yellow and weaker vortices are colored by less-opaque blue, with the opacity and color scales displayed at the bottom of the plate.

The streamwise vortex pairs are visible beginning at $t=$ 62.5 as the ghostly white streaks. Streamwise vorticity arises from both direct baroclinic generation of streamwise vorticity and from tilting spanwise shear vorticity into the streamwise direction. The former source dominates at early times, while the latter dominates at later times. This supports the observations by Fritts et al. (1994, 1996a) that the major sources of instability kinetic energy are a conversion from eddy (i.e., 3D) gravitational potential energy at early times and a conversion from shear kinetic energy at later times. The influences of the streamwise vortices on the isosurfaces of potential temperature can be seen in Plate 1 in the panels at $t=62.5$ and 65, where downward and upward displacements of the potential temperature surface occur inside and outside vortex pairs respectively.

In the next phase of the evolution, the streamwise vortices stretch and tilt the spanwise vorticity of the shear (both the background shear and the shear of the wave itself). The lower panels of Plate 1 show the enstrophy of the full domain at $t=65$ and $t=70$. Two views of each are shown: from positive $x$ and above on the left, and from below on the right. High values of enstrophy are bright pink and opaque and low values are blue and nearly transparent. Considering $t=65$ first, we see that there is significant enstrophy which is not represented in $\lambda_{2}$ (compare the upper right enstrophy panels of Plate 1 with the upper right panel of Plate 2). This is because most of the enstrophy in Plate 2 lies in sheets, and does not have the rotational character required to appear in $\lambda_{2}$. Indeed, the enstrophy shown in Plate 1 at $t=65$ has vorticity predominantly in the spanwise direction, and represents the vorticity of the total shear. By $t=70$, however, a comparison of the enstrophy and $\lambda_{2}$ images (lower right panel of Plate 1 and the second right panel of Plate 2) reveals that the majority of the enstrophy is now associated with the vortex tubes.

Prior to the appearance of streamwise vortices, the spanwise vorticity due to the shear has no spanwise structure. It varies only in the streamwise and vertical directions. The streamwise vortices created by the convective instability then advect and stretch the spanwise vorticity in their flow. Note, in particular, the shape of the enstrophy in the upper left panel of the lower set in Plate 1 at $t=65$. The areas of brightest pink are strong regions of enstrophy with vorticity in the positive spanwise direction that have been advected downward by the convective rolls. As they are advected downward, their spanwise vorticity is stretched because the flow of the convective rolls diverges in the spanwise direction and so contributes to intensification via stretching. This amplifies the enstrophy advected downward over the background enstrophy, and makes the amplified regions thinner since the flow of the convective rolls is convergent in the vertical direction. Interspersed with the regions of strong downward-advected enstrophy are regions of enstrophy that have been advected upward and have also been amplified by stretching. These regions, which have negative spanwise vorticity, are weaker than the downward-advected regions because the mean and wave shears are of opposite sign at this phase of the wave. The enstrophy at each wave phase is concentrated in sheets of spanwise vorticity with streamwise extents of roughly 10 20 sheet thicknesses, and spanwise widths of roughly 6 sheet thicknesses.

The enstrophy sheets are unstable to a spanwise-localized (3D) Kelvin-Helmholtz $(\mathrm{KH})$ instability and roll up into a series of vortex tubes. The beginning of one of these roll-ups is visible in the upper right enstrophy panel of Plate 1 in the topmost vortex sheet on the left side of that panel. That sheet is rolling up into four vortex tubes, all of which are curved in the same manner. This curvature is easily explained by noting that the edges of the original sheet are curved upward, and so the ends of the rolled-up tubes lie above their centers. The mean shear flow advects the tube ends downstream and rotates the curvature of the tubes. At the later time $t=70$, all the sheets have rolled up into tubes. Noting that the dynamical timescale of a shear layer is $t \sim(d U / d z)^{-1}=\omega^{-1}$ which is roughly $t \simeq 0.2$ for a typical vortex sheet, we see that the roll-up of the sheet takes about 10 shear timescales. The resulting vortex tubes are clearly visible in Plate 2 showing the $\lambda_{2}$ eigenvalue. This KH instability (which we call the secondary instability as it follows and is triggered by the primary convective instability), then, is a distinct and robust feature of the flow; it rolls all the vortex sheets into vortex tubes which then form intertwined horseshoe-shaped vortex loops. In Plates 1 and 2, note that the enstrophy and vortex fields are advected toward larger $x$ (to the right) by the mean streamwise motion so that the fields are translated by $\sim 1 / 3$ of the domain length from $t=65$ to 70 . Because of the streamwise shear flow, however, the advection is weaker at lower levels, with the lower structures moving more slowly toward larger $x$. Additional discussion of this evolution and of the $\mathrm{KH}$ structures that arise is provided by Andreassen $e t$ 

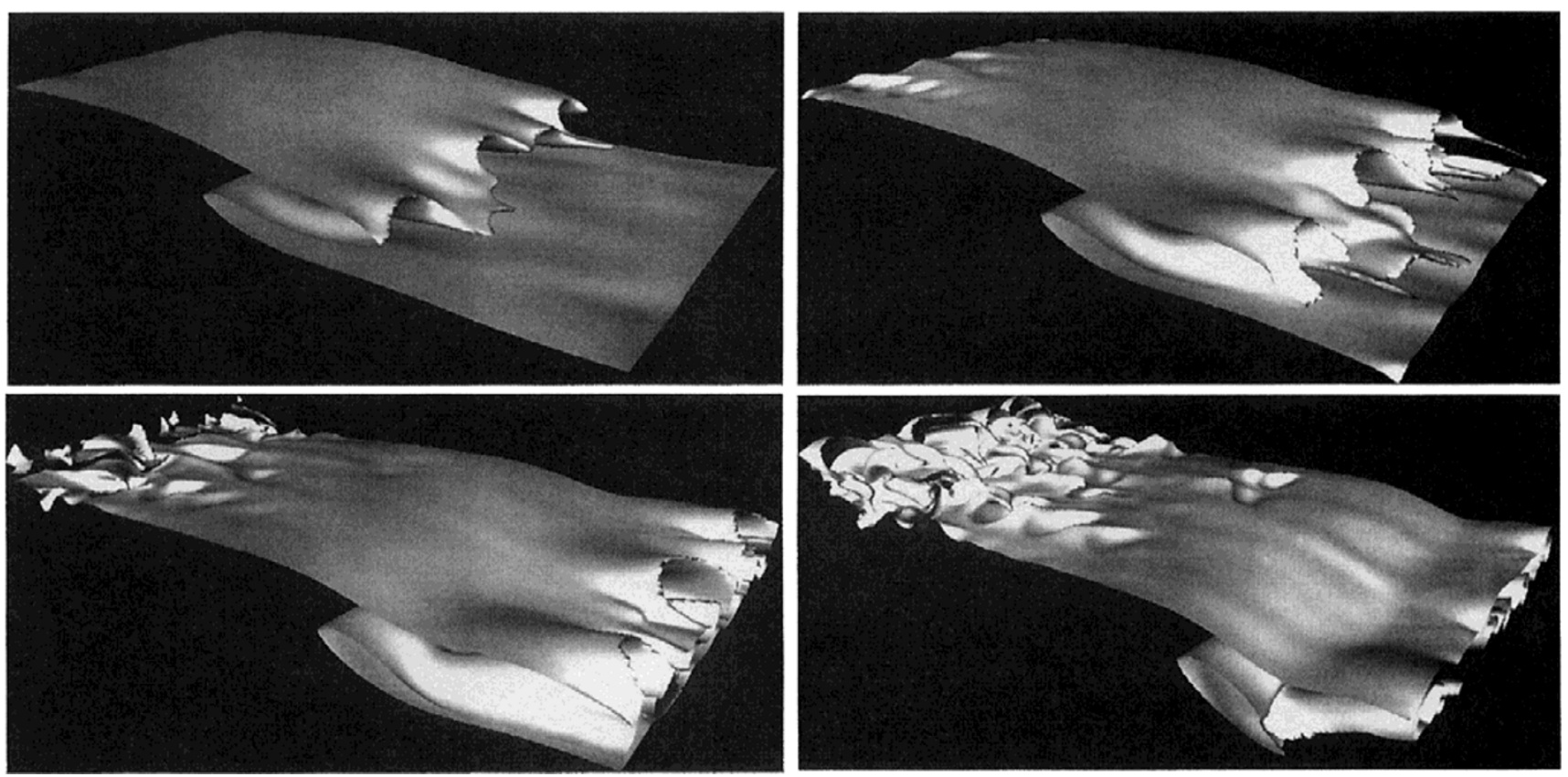

(a)
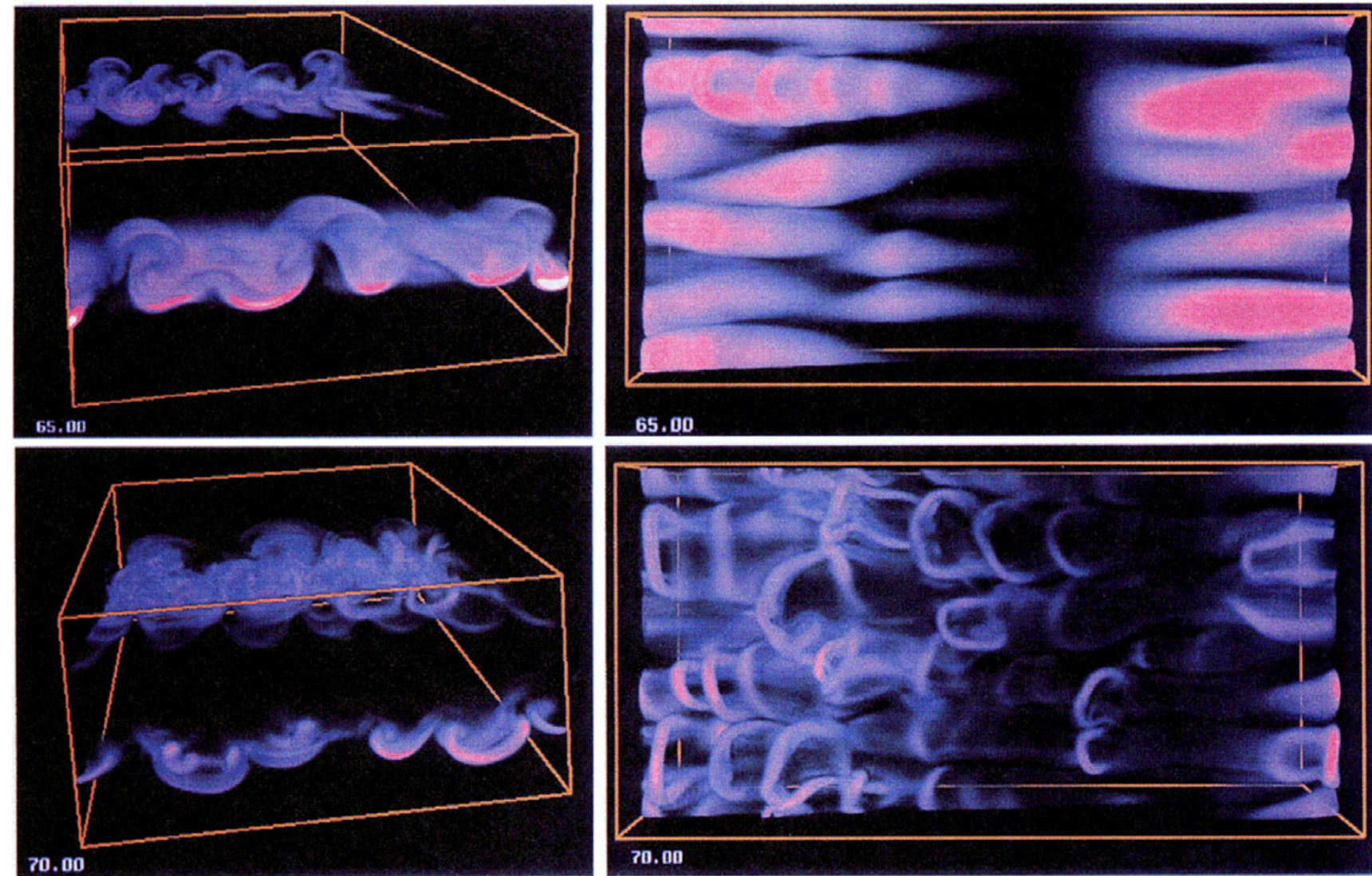

(b)

Plate 1. (a) Isosurface of potential temperature with $\theta=2.97$ within the region of internal gravity wave breaking at $t=62.5,65,67.5$, and 70 . (b) Volume renderings of enstrophy viewed from below with positive $x$ (streamwise) to the right in the right panels, and viewed from positive $x$ and above in the left panels. Top panels show $t=65$ and bottom panels show $t=70$. Enstrophy is shown with large values opaque and pink and weak values transparent and gray. 

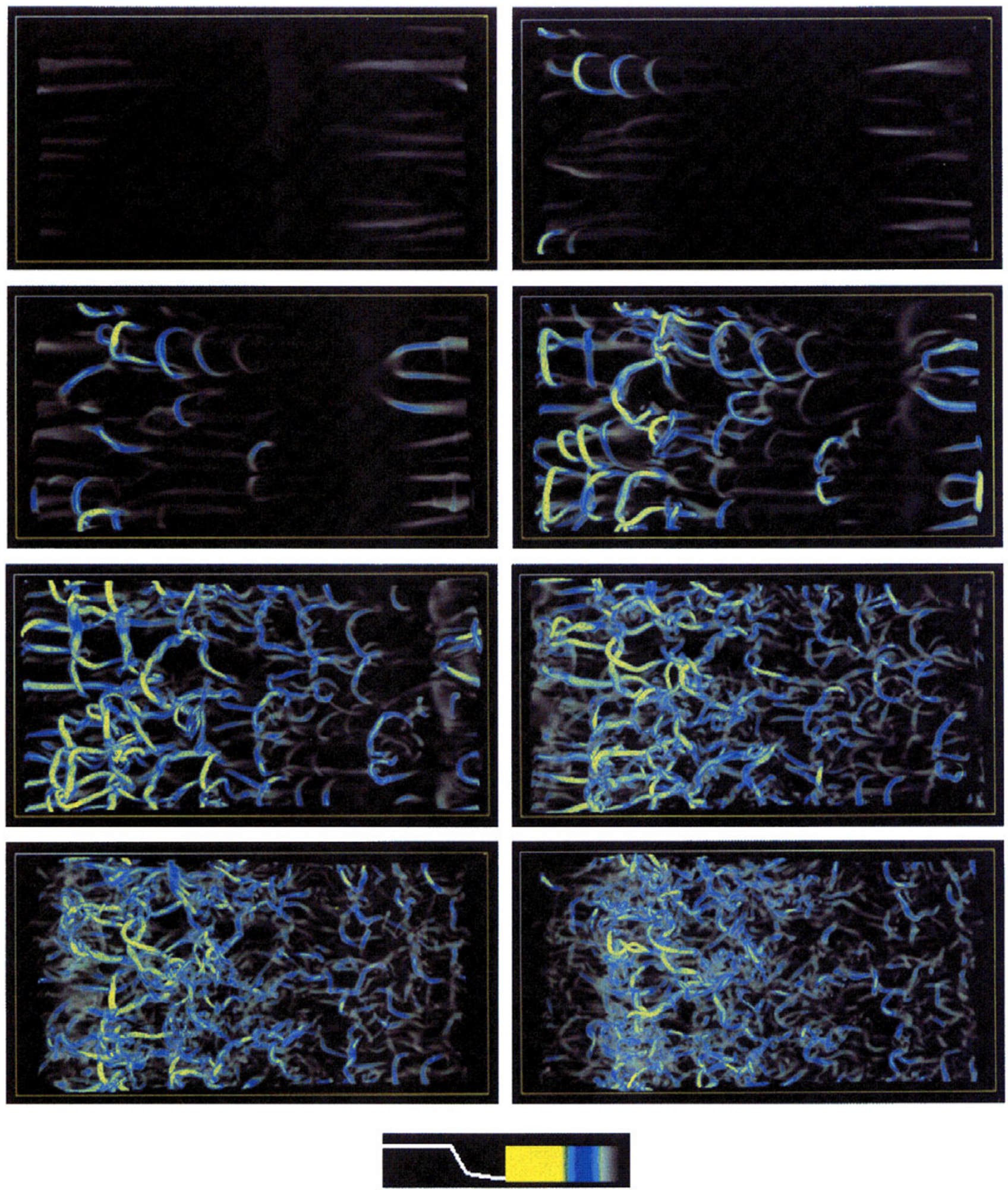

Plate 2. Volume renderings of $\lambda_{2}$ from below with positive $x$ to the right at $t=62.5,65,67.5,70,72.5,75,77.5$, and 80 left to right and top to bottom. Color and opacity scales are shown at the bottom. The opacity scale extends from transparent (bottom) to opaque (top); both scales show negative $\lambda_{2}$ with zero at the right.

al. (1998). These dynamics are also similar in important respects to the evolution of a sheared boundary layer in which streamwise vortex structures have been observed to elevate and stretch the spanwise vorticity, leading to an inflectional velocity profile and secondary roll-up of the intensified vortex sheets (Kline et al., 1967; Landahl and Mollo-Christensen, 1992).

The net result of the primary convective and secondary dynamical $(\mathrm{KH})$ instabilities is a collection of intertwined vortex loops having counter-rotating streamwise "legs" in- 
clined along the phase of the wave motion and having centers with positive spanwise vorticity. Successive loops (toward negative $x$ or upstream) have their streamwise legs above and within the adjacent downstream (toward positive $x$ ) loops. The result is a complex vorticity field having many sites where vortices having approximately parallel, antiparallel, or orthogonal alignments occur in close proximity and interact strongly. The vortex loops bear a close resemblance to the "horseshoe" and "hairpin" vortices that arise in turbulent boundary layer flows (Acarlar and Smith, 1987; Robinson, 1991; Sandham and Kleiser, 1992), in stratified and sheared turbulence (Gerz et al., 1994), and in rotating shear flows (Metais et al., 1995). The dynamics of these structures, then, may have implications for the evolution of a broad class of flows.

The series of intertwined vortex loops arising from initial convective and secondary dynamical instabilities condition the flow for a rapid subsequent evolution because of the close proximity of adjacent vortices. The further evolution of this vortex field is displayed in the lower four panels of Plate 2 at $t=72.5,75,77.5$, and 80 . Though the time required for this further evolution of the flow spans only $\sim 0.7 T_{\mathrm{b}}$, the increase in flow complexity is dramatic. The vortex loops present at $t=70$ are fragmented into pieces, and new smaller vortex tubes continue to be formed (small blue and gray wisps in Plate 2). At later times, apart from a large-scale correlation with the phase of the original gravity wave, the small-scale flow is almost isotropic, and has little evidence of its initial state. The vortices lose their initial streamwise and spanwise orientation and structures appear at significantly higher wavenumbers (by a factor of 2 to 3 ). Additionally, the maximum vorticity and enstrophy at small scales of motion increase throughout the simulation, both because of the initial cascade from larger to smaller scales and because enstrophy is larger at the smaller scales within an inertial range of turbulence. The dynamics accounting for this further evolution toward a more chaotic and isotropic vortex field and smaller scales of motion are the subject of the remainder of this paper.

\section{Primary Vortex Dynamics}

We have identified several processes that appear to be important in the evolution toward smaller scales and increasing isotropy. This section addresses the vortex dynamics that we judge to be key to the transition to and the cascade within a turbulent flow. Other vortex interactions that participate in the evolution, but are believed to be less important, are described in the following section. Several of these processes can be seen to occur in the series of closeup images of the vortex field extending from $t=70$ to 75 in Plate 3 .

\subsection{Orthogonal vortex stretching}

The key element in vortex intensification on short time scales is vortex stretching, either of the ambient shear field by localized vortices (or vice versa) or of localized vortices by other localized and closely spaced vortex structures. For this process to be effective, the local component of vorticity, $\omega_{i}$, and the diagonal component of the strain tensor, $S_{i i}$, must contribute a source, $\omega_{i} S_{i i}$, which exceeds other baroclinic, compressional, and viscous contributions. This stretching source is particularly strong when the vorticity lines of two neighboring vortex structures are nearly orthogonal. Phys- ically, the stretching is due to a flow divergence along the vorticity. Because of the geometry of the flow, vorticity is stretched on the upstream side (relative to the point of closest approach) of an orthogonal vortex, and scrunched (negative stretching via a convergent flow) on the downstream side. This mutual stretching (and scrunching) is illustrated schematically, with vortex thickness indicating strengthening and dashed lines denoting weakening, in the first series of diagrams in Fig. 1.

Noting that the vorticity lines of the vortex sheets are predominantly spanwise (vertical in Plate 2) and that the vorticity lines of a vortex tube are roughly along the tube, we see that there are indeed numerous sites where vortex structures in Plate 2 have approximately orthogonal alignments and undergo local intensification. When the stretching involves a vortex sheet, roll-up and tube formation can result, as discussed above. A good example of such orthogonal vortex stretching can be seen in the upper central part of the images at the first three times (left three panels, top to bottom) displayed in Plate 3. Here (as seen from below), a vortex having positive spanwise vorticity (directed down toward positive $y$ ) overlies a streamwise vortex having negative streamwise vorticity (toward the left, or negative $x$ ). Based on the arguments above, we should expect that the streamwise vortex causes divergent/convergent motions (stretching/scrunching) along the overlying (at smaller $z$, seen from below) spanwise vortex, leading to an intensification with time of the portion of the spanwise vortex toward positive $y$ (below) and depletion of the vortex at negative $y$ (above) relative to the streamwise vortex. In the same manner, the spanwise vortex stretches/scrunches the streamwise vortex toward negative/positive $x$ where it underlies the spanwise vortex. The same effect can also be seen in the upper left panel of Plate 4, which focuses on only this single vortex at an intermediate time.

An important consequence of this mutual stretching is the preferential amplification of the orthogonal vortex structures, causing these structures to become most prevalent as the vortex field evolves. Indeed, these " $x$-patterns" appear as frequent and persistent features in the vortex evolution and can be seen surviving to late times relative to other features of the flow in Plate 2. Such " $x$-patterns" were observed by Boratav et al. (1992) and Pumir and Siggia (1990) to lead ultimately to anti-parallel vortex alignments at late times in an unstratified flow. However, late-time anti-parallel alignments are not observed in our flow, perhaps as a result of the more viscous nature of the vortex dynamics in our simulation.

\subsection{Vortex sheet and tube formation}

Formation and intensification of vortex sheets and their subsequent roll-up into vortex tubes were identified as initial steps in the transition to and within turbulent flow by Betchov (1957) and Lundgren (1982). Our results and the previous discussion of Plate 2 show that these processes also occur in a stratified fluid and play a continuing role throughout the flow evolution. Sheet roll-up occurs on sheets which have been intensified by vortex stretching from the flow of a neighboring orthogonal vortex. The roll-up proceeds by analogy with the $2 \mathrm{D} \mathrm{KH}$ instability of an infinite vortex sheet studied by many authors or the 3D KH sheet instability described in the previous section. 

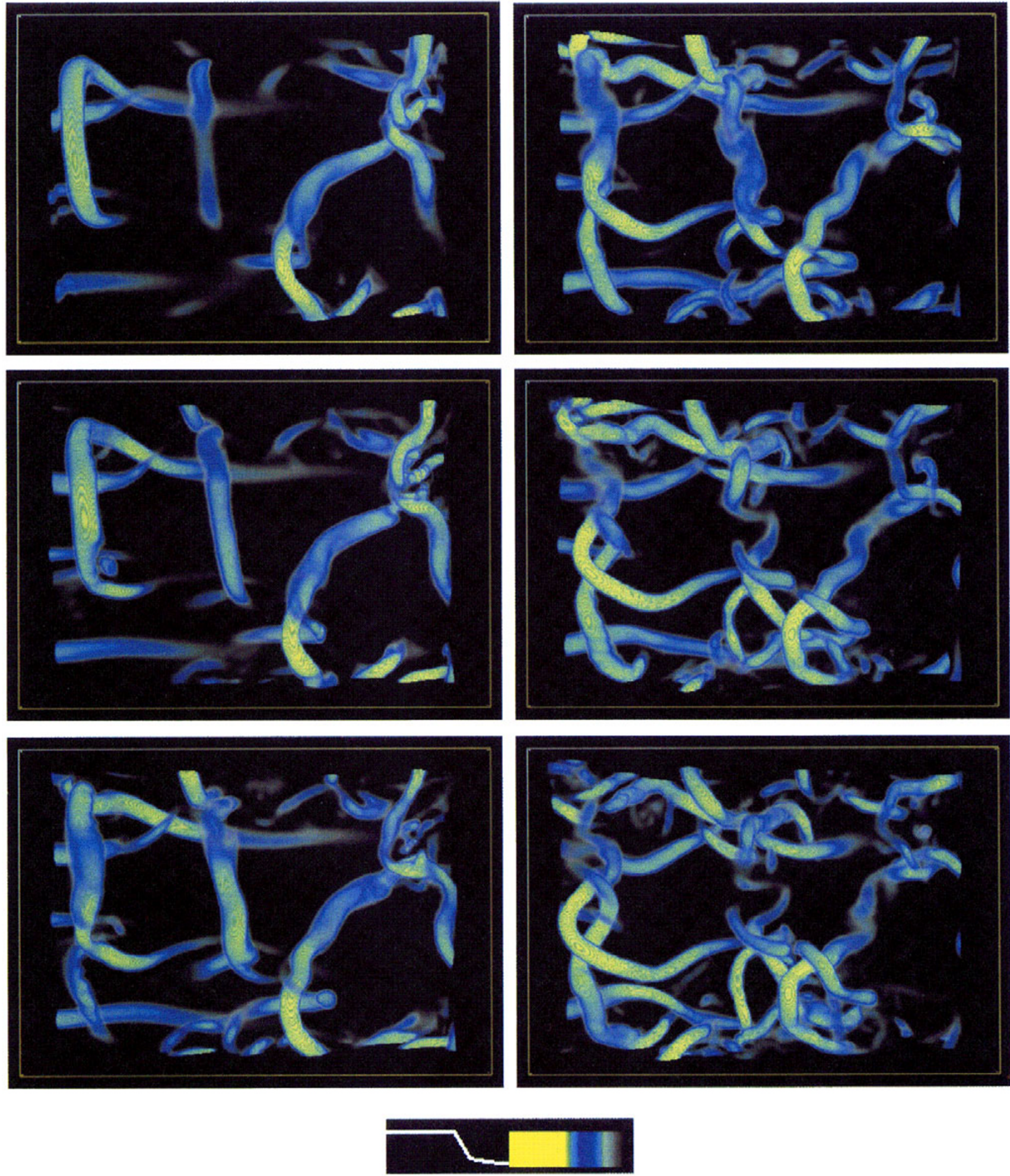

Plate 3. As in Plate 2 for a subdomain at the upper left of the full domain including $\sim 1 / 3$ of the domain in $x$ and $1 / 2$ of the domain in $y$. Times are $t=70$, $71,72,73,74$, and 75 from top to bottom and left to right. Color and opacity scales are shown at the bottom.

Examples of vortex sheet formation and roll-up can be seen in the temporal evolution displayed in Plate 3, where small vortex segments can be observed to form and intensify with time in close proximity to the stronger vortex tubes. Specific examples occur 1) at the lower left end of the spanwise vortex tube just discussed at early times, 2) in an identical fashion at the lower left end of the vortex tube at the left of the images at early times, and 3) at smaller scales adjacent to the stronger vortices at later times. The first of these examples is again displayed clearly in the three upper images showing the temporal evolution of the spanwise vortex tube in Plate 4. In each case, vorticity vectors of the tube and adjacent sheet are approximately orthogonal. As the evolution progresses, the vortex sheet intensifies due to stretching by the overlying 

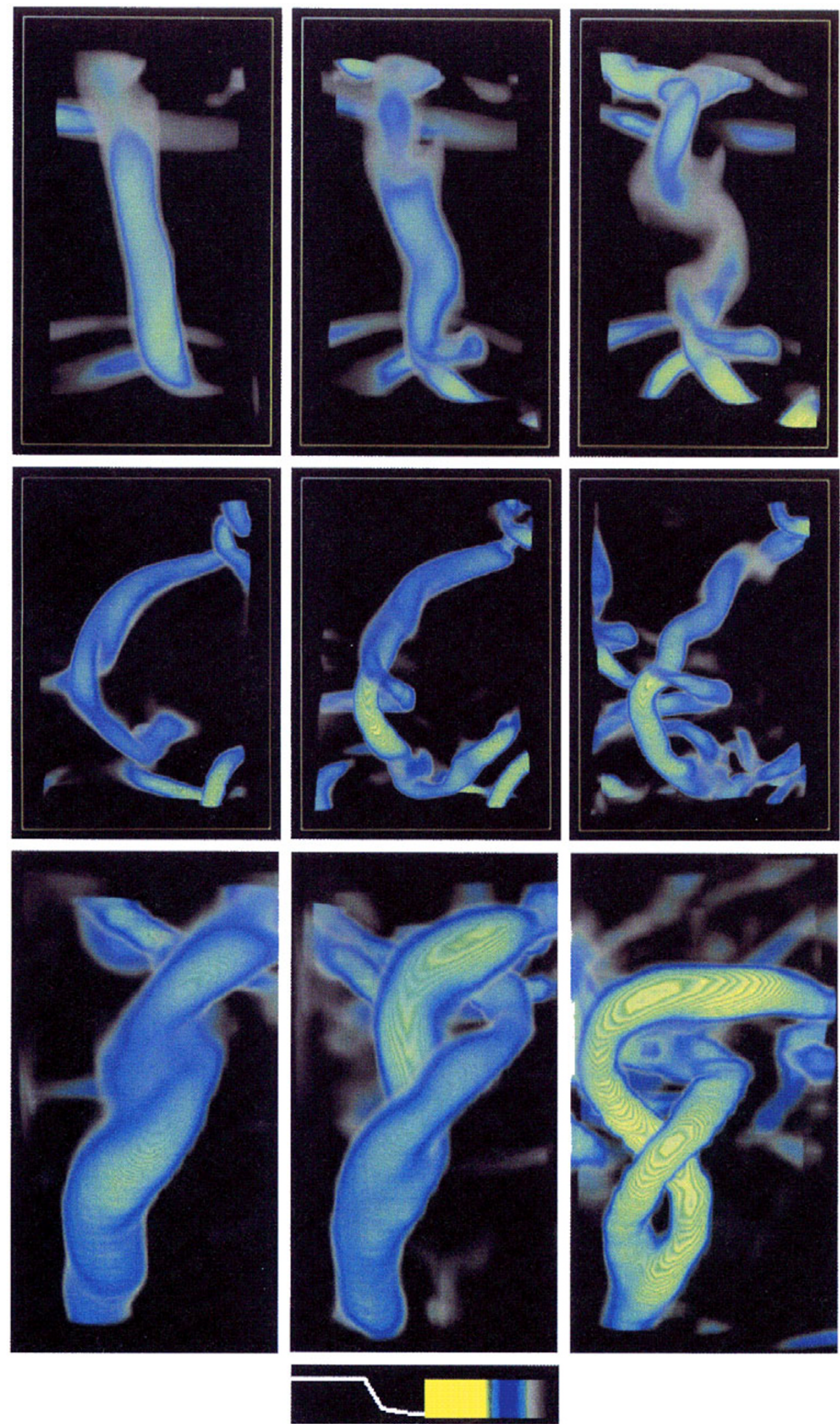

Plate 4. As in Plates 2 and 3 for the spanwise vortex at the center of the images in Plate 3 at $t=71.5,72.5$, and 73.5 (top), the curved vortex at the lower left of the images in Plate 3 at $t=69,71$, and 73 (middle), and the streamwise vortex just above the left center of the last two images in Plate 2 at $t=76$, 78 , and 80 (bottom). Color and opacity scales are shown at the bottom. 
a)
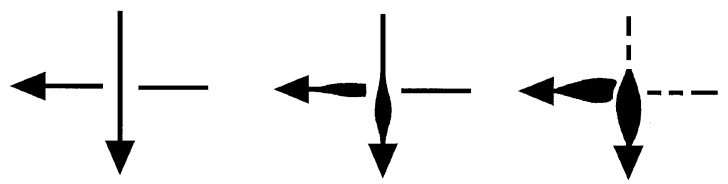

b)
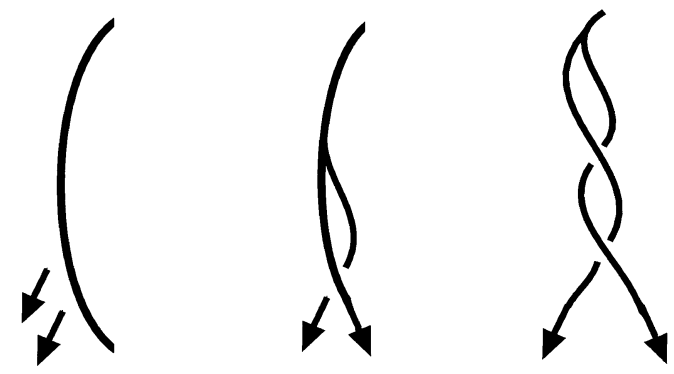

c)
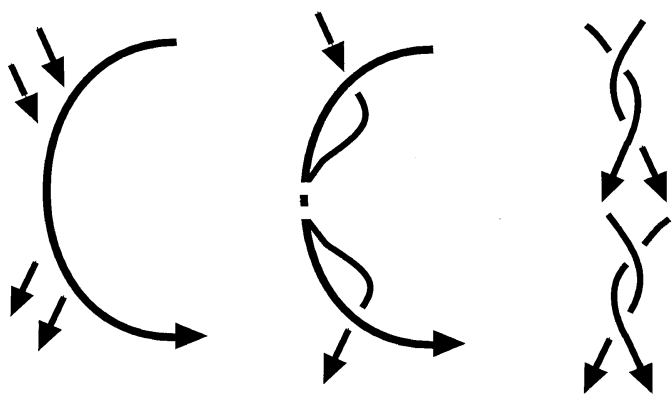

Fig. 1. Schematics of three of the primary vortex interactions which account for the dominant vortex structures and drive the cascade of enstrophy toward smaller scales. The upper series illustrates the differential stretching and scrunching of orthogonal, mutually interacting vortices leading to intensification and long-lived " $x$-pattern" structures. The central series illustrates the intensification of a weak vortex sheet, its attachment to a stronger vortex tube, and the subsequent twist wave amplification, propagation, and fraying under unstable conditions (see text for details). The lower series illustrates the impact of mode-two twist waves initiated at different sites on a vortex tube that propagate toward each other and result in the unraveling and breakup of the vortex tube.

(at smaller $z$ ) vortex tube. Continued stretching by the vortex tube intensifies the vortex sheet locally, until it rolls up via a local KH instability and forms a tube of comparable intensity with that accounting for the stretching.

\subsection{Mode-zero twist waves}

We noted earlier that the vortex loops arising from the secondary $\mathrm{KH}$ instability tend to fragment into pieces. One of the causes of this fragmentation is the presence of modezero (azimuthal wavenumber $m=0$ ) twist waves of large amplitude. Examples of mode-zero twist waves can be seen on many of the spanwise-aligned vortices in Plate 2, more clearly in the two left-most spanwise vortex tubes in the first several images in Plate 3, and in the sequence of three images at the top of Plate 4 . In each case, the mode-zero twist waves are initiated through the stretching/scrunching of a vortex by a near-orthogonal neighbor via the " $x$-pattern" interaction. In the case of many of the spanwise vortices, mode-zero twist waves appear to be excited at both ends of the vortex simultaneously, to propagate inward from the sites of excitation, and to jointly cause a very strong axial convergence which weakens the vortex to such an extent that its center is obliterated. This process is responsible for the inward motion of the depleted (weaker) vorticity from either end of the spanwise tube seen to occur in the top left and center panels of Plate 4. The third panel in this sequence reveals the consequence of the collision of two large-amplitude mode-zero twist waves which, with superposed axial divergence fields, destroy the vortex core. Given the prevalence of mode-zero twist waves in our simulation and the consequences for vortex breakup, we conclude that this is a key mechanism in driving the evolution toward smaller scales of motion.

The propagation and characteristics of twist waves were first described by Kelvin (1880). Similar dynamics of a vortex tube were recently discussed by Melander and Hussain (1995) and Schoppa et al. (1995) in association with vortex core dynamics, while Arendt et al. (1997) recognized the applicability of twist waves in the interpretation of our wave breaking vortex dynamics. In general, twist waves are dispersive traveling waves on vortex tubes. The axisymmetric (mode-zero) modes propagate by twisting the vortex lines of the tube, thereby creating an axial flow. The axial flow changes the enstrophy of the tube by stretching/scrunching. This, in turn, changes the rotation rate of the tube which then changes the twist. From this point of view, the event shown in the top panels of Plate 4 is a standing axisymmetric twist wave on a vortex tube excited by the initial state of the tube, including its proximity to the streamwise tubes underlying it (Arendt et al., 1997). The wave is of such large amplitude that it irreversibly depletes the middle of the vortex tube during its minimum.

\subsection{Mode-two twist waves}

Another type of twist wave that plays a major role in the fragmentation of vortex loops is a mode-two (azimuthal wavenumber $m=2$ ) wave. These waves are generally excited where a vortex tube is perturbed near its end by another vortex structure (either a tube or sheet). The clearest and most long-lived examples of mode-two twist waves in our numerical solution arise due to upstream perturbations of the more axially-uniform streamwise vortex tubes. Two of these waves are seen in the upper left portions of the lower right images shown in Plate 2; other examples are seen to arise on the spanwise vortices seen in Plate 3 at later times. The evolution of the mode-two twist wave seen in the lower right panel of Plate 2 is shown magnified in the lower panels of Plate 4, again viewed from below, where now the $x$ and $y$ directions are down and to the left.

In each case, a streamwise-propagating $m=2$ twist wave with a small amplitude is excited on the vortex tube; the propagation of the wave is not seen since the domain displayed is adjusted to track it. The waves grow until they are sufficiently large to unravel the vortex tube into a pair of intertwined helices; a schematic of this is shown in Fig. 1(b).

It is useful to examine how a mode-two twist wave propagates. Essentially, such a wave is propelled by self-advection, i.e., the vortex tube induces a velocity field, and is then advected by it. This is a common effect in vortex dynamics. A simple example is that of a vortex ring which is propelled in a direction perpendicular to its plane by its own velocity field. In the case of an $m=2$ wave, each helix of the pair induces a velocity field with both an azimuthal rotating component and an axial component. The axial component 


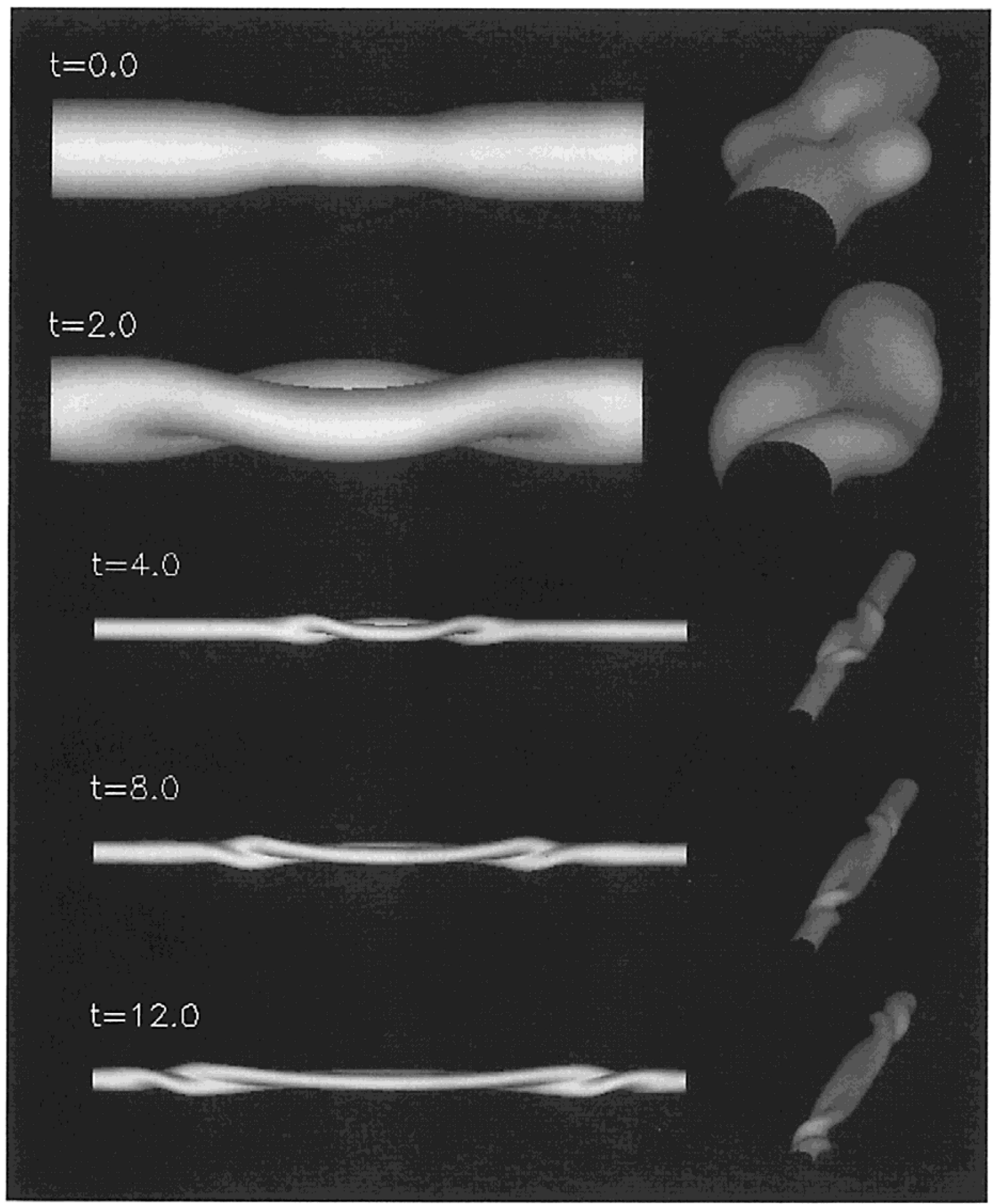

Plate 5. Analytic mode-two twist waves excited by a small-amplitude, axially-Gaussian $m=2$ perturbation at $t=0$. Times are in units of the circulation time, the upper two images focus on the source region, and the latter three images display more of the vortex tube to exhibit the wave packet structure. The vorticity is to the left in the left panels, the right panels show the views from the left end of the tube, and the wave amplitude has been exaggerated for ease of visualization.

propels the wave by advecting the helices. For example, the tube shown in the bottom panels of Plate 4 has vorticity vectors pointing downward. Given the sense of the helices of the wave, it is easy to show (using the Biot-Savart law or simply the right-hand rule) that the axial velocity is downward, and so the wave propagates downward. At the head of the wave packet, where the tube is unsplit, the axial flow of the approaching double helices precedes the helices themselves The axial flow decays with distance from the helices, and so it is convergent along the axis in front of the wave. If the flow converges axially, it must diverge radially; this radial divergence splits the vortex tube into the two component helices.

An important consequence of the self-advected propaga- 
tion of an $m=2$ wave is that the direction of propagation is determined by the direction of the vorticity in the tube and the sense of the helices of the wave packet. These two components determine the direction of the axial flow, and hence the direction of propagation. The mode-two twist wave seen at the upper left at $t=75$ in Plate 2 has the opposite sense of helicity and vorticity and therefore also propagates in the positive- $x$ direction.

Another example of a mode-two twist wave is seen at the left edge of the latter images (right panels) in Plate 3. Consider the $\lambda_{2}$ evolution. After the dominant spanwise vortex loop forms, structures which underlie it and which are initially weak intensify (see the lower end of the tube at the left edge of the lower left panel) and appear to attach to the spanwise vortex loop. This process initiates a mode-two twist wave which propagates along and unravels the spanwise vortex tube. Equivalent dynamics are also initiated at the opposite end of this same vortex tube, but here the helicity and direction of propagation are reversed. The consequence in this case is a spanwise vortex tube that decays via unraveling from both ends rather than bursting due to mode-zero twist wave propagation. These influences on the spanwise vortex tube are displayed schematically in Fig. 1(c). Such events are very common throughout our simulation. Similar unraveling events are also observed in the laboratory (Cadot et al., 1995) and are likely also a consequence of large-amplitude, modetwo (or higher $m$ ) twist waves. These dynamics are also discussed in greater detail by Fritts et al. (1998).

Though the twist waves discussed above are of large amplitude and have clearly nonlinear effects (i.e., they destroy the vortices on which they propagate), their propagation can be understood in terms of linear theory. To exhibit this behavior, we describe analytically the mode-two twist wave structure on a vortex of constant vorticity and finite radius (see also Arendt et al., 1997) which arises from a smallamplitude, axially-Gaussian, mode-two perturbation. The resulting wave propagation is displayed in Plate 5 (with amplitude exaggerated), where now the times are in units of the circulation time of the vortex. The vorticity of the tube is to the left; hence two wave packets are excited having opposite helicity and direction of propagation, just as observed in our simulation results and discussed above. This can be understood by noting that right-handed helicity implies an induced axial motion and self-advection in the direction of positive vorticity, with the opposite sense of propagation for the opposite helicity. This accounts for the correlation between the vorticity and helicity seen in the latter panels of Plate 2, where both mode-two waves are seen to propagate toward positive $x$. Finally, we note that the mode-two twist waves exhibit dispersion, with the smaller-scale waves apparently propagating with larger group velocities. These and other details, including the radial structure of such waves, are discussed further by Arendt et al. (1997).

\section{Secondary Vortex Dynamics}

In this section we discuss several processes which play clear roles in the evolution of the vortex field due to wave breaking, but which appear to be of minor importance to the cascade of energy and enstrophy to smaller scales of motion. These include vortex reconnection, mode-one twist waves, and vortex pairing. Of the three, reconnection is easily the most pervasive, but perhaps the most difficult to quantify. Mode-one twist waves, in contrast, are easily seen in the numerical solution, arise from perturbations to vortex tubes, and can be described simply in terms of linear theory. Finally, vortex pairing arises when two spanwise loops are close to each other and proceeds in a manner analogous to such pairing events in 2D flows.

\subsection{Vortex reconnection}

Reconnection events fall into two broad classes. The first are those described by Kida and Takaoka (1994) in which vortex tubes undergo attachment without requiring viscous reconnection of vortex fieldlines. An example of this sort of event occurs at the lower left portion of the domain displayed in the top sequence of images in Plate 4, where the initially weak tube amplifying through the " $x$-pattern" interaction intensifies and attaches to the spanwise vortex. Examples of this sort are common in our simulation and are easy to spot through a close examination of Plate 2.

The second, and more interesting, class of reconnection events, those in which vortex fieldlines reconnect, has been a topic of detailed study (Pumir and Siggia, 1990; Boratav et al., 1992; Shelley et al., 1993). An example of such an event occurs at the upper right in the first three $\lambda_{2}$ images in Plate 3 (left panels). Here, a vortex loop originating on one vortex sheet (see the strongest vortex at the upper left in the second left panel of Plate 2) begins to be distorted by a vortex arising on an adjacent sheet (below and to the left). By $t=70$ (top left image in Plate 3), the initial vortex loop is strongly kinked, but still intact. Thereafter, its close proximity to the underlying vortex causes stretching and scrunching due to their approximately orthogonal orientation. This enhances the leftmost part of the loop and weakens the portion to the right just where the vortex loop itself is undergoing a breakup, enabling viscous alignment and attachment of the fieldlines among formerly discrete vortices. The result is the new sinuous vortex seen entering the domain at the top near the right edge (lower left panel of Plate 3 ) and exiting below the midpoint on the right edge.

\subsection{Mode-one twist waves}

A final type of twist wave present in our numerical solution is the mode-one (azimuthal wavenumber $m=1$ ) twist wave. This wave perturbs a vortex tube into a single helix and propagates through self-advection, as in the mode-two case. A clear example of a mode-one twist wave is on the vortex slanted at $\sim 45^{\circ}$ at the lower right of the expanded images in Plate 3. At the earliest time shown, $t=70$, the tube is largely unperturbed, but is frayed near its lower end. At later times, the tube is bent into an increasingly wiggly pattern. A further expanded view of the evolution of this tube spanning $t=70$ is shown in the central three panels of Plate 4 . These images show a vortex exhibiting left-handed helicity and having positive spanwise (or downward) vorticity.

The mode-one twist wave is apparently excited by the close orthogonal neighboring vortex underlying it at its lower end. The flow of this orthogonal neighbor bends the tube initially into a single wiggle, and the self-dynamics of the tube transforms the single wiggle into a full helix thereafter. Curiously, the wave does not seem to propagate. Rather, it appears to be a standing wave made stationary by the stationary forcing 


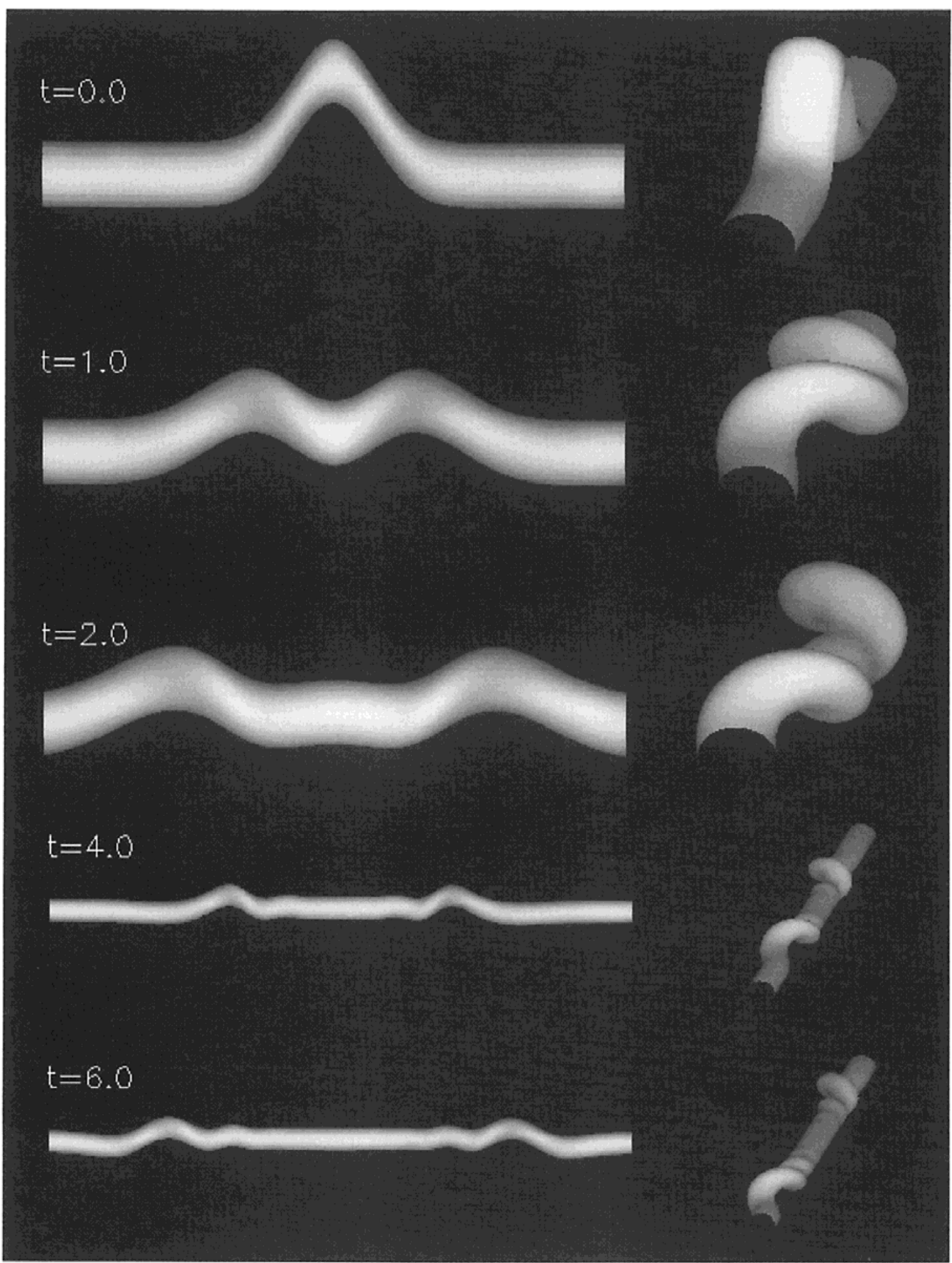

Plate 6. As in Plate 5, but for a mode-one twist wave.

due to the neighboring orthogonal vortex. Alternatively, the mode-one twist wave may be a true stationary wave, as such waves do occur (Saffman, 1992).

Other mode-one twist waves are also observed to be excited in our simulation, including two (apart from that discussed above) in the third image on the left in Plate 2, at the lower edge of the domain about $1 / 3$ of the distance from left to right and at the top center, both of which have largely spanwise alignment. Expanded images at later times reveal other examples. Thus, the mode-one twist waves comprise a significant feature of the turbulent dynamics in our simulation. They are not judged central to the vortex and turbulence evolution, however, because they appear not to contribute strongly to the breakup of larger vortices or the cascade to 
smaller scales of motion.

As for the mode-two twist waves discussed above, we provide here an analytic example of a mode-one twist wave on an idealized vortex in order to display the propagation and dispersion of such waves. We again assume a smallamplitude, axially-Gaussian perturbation, which in this case represents a deflection of the tube. This perturbation induces self-advection of the tube displacement which excites mode-one twist waves propagating in each direction along the tube, with the direction determined by the self-advection due to the axial component of the induced velocity field (see Plate 6). The vorticity of the tube is to the left, resulting in the twist wave having right-handed helicity propagating to the left (in the direction of positive vorticity) and the twist wave having left-handed helicity propagating in the opposite direction. Also seen clearly in the panels at later times (again expressed in units of the circulation time of the vortex) is the dispersive character of the mode-one waves, with larger scales having larger group velocities along the tube. Note that this is opposite to the dispersion noted with the mode-two twist waves above. Further discussion of modeone twist wave structure and behavior is provided by Arendt et al. (1997).

\subsection{Vortex pairing}

Vortex pairing, the mutual interaction of two parallel vortex tubes, is generally understood on the basis of 2D laboratory and numerical studies. This process is often observed at a sheared interface in fluids that are homogeneous or have a density gradient confined to the shear layer (Scotti and Corcos, 1972; Patnaik et al., 1976; Koop and Browand, 1979; Fritts, 1984; Thorpe, 1985; Metcalfe et al., 1987), and can be viewed as a degenerate resonant triad interaction between the primary KH billow and its subharmonic (Davis and Peltier, 1979). Pairing is less pronounced in a fluid that is stratified away from the shear layer, however, for two reasons. First, the mean state Richardson number required for linear subharmonic instability, and hence for efficient subharmonic excitation, is much less than for the primary instability (Drazin, 1958). Second, subharmonic excitation at larger Richardson numbers is suppressed because the subharmonic has propagating rather than evanescent character away from the shear layer and therefore radiates away as it is excited (Fritts, 1984). As a result, such pairing events appear not to be observed in the stratified atmosphere and oceans.

We observe several cases of vortex pairing in our results. Generally they resemble their 2D analogs, with the vortices approaching closely, mutually advecting, and wrapping around each other. Pairing is different in 3D in two respects. First, the vortex sheets are localized in the spanwise direction, and the resulting tubes are as well. At later times, the tubes are curved into loops with the streamwise extensions of the loop legs coming close together. So, the pairing proceeds between adjacent loops, with one loop (typically the upstream loop in our simulations) being advected over and within the other loop, and at the same time being weakened due to scrunching. The pairing then resembles leap-frogging vortex rings.

The second difference in $3 \mathrm{D}$ is that other processes often occur on faster timescales within the loops and disrupt them before the pairing can proceed very far. For example, on some occasions we observe twist waves (both mode-zero and two) fragmenting and unraveling the vortices while they are pairing. Pairing is less disrupted if it takes place at small scales; presumably, twist waves are heavily influenced by viscosity at these scales (as is the pairing) and are unable to compete with the pairing. Furthermore, in a previous simulation employing lower resolution and $25 \%$ greater dissipation, the twist waves at this stage of the simulation were somewhat suppressed and the vortex pairing proceeded with less competition. Finally, it is possible that the pairing events facilitate other vortex interactions (i.e., twist waves) by driving the more intense vortex structures closer together. Pairing of adjacent vortices is seen at several sites in our simulation, notably between the strong vortex and the weaker and smaller vortex within it and at smaller $x$ (to its left) at the upper left in the second left panel of Plate 2. A second example occurs at the lower left of the third left panel of Plate 2, but is not well resolved in time and has largely decayed by the next image. These dynamics are not displayed separately because of our judgement that they are of less importance in the evolution of the vortex field; however, these and other twist wave dynamics are discussed at greater length by Fritts et al. (1998).

\section{Enstrophy Spectra and Cascade}

To illustrate the enstrophy evolution accompanying wave field instability and the subsequent cascade toward smaller scales of motion more quantitatively, we now examine the temporal development of the total enstrophy spectra with streamwise and spanwise wavenumbers $k_{x}$ and $k_{y}$. These are shown in Fig. 2 at $t=60,65,70,75$, and 80. Note that the $k_{y}$ spectra are corrected for the difference in the $x$ and $y$ domain size to permit comparison of spectral amplitudes at the same scales in each direction. These spectra exhibit clear differences at early times $(t=60$ and 65). The $k_{y}$ spectra (dash-dotted and dashed lines) exhibit a series of discrete peaks corresponding to the scales at which the dominant spanwise instabilities (i.e., the streamwise convective rolls) arise (wavenumbers 4, 10, and 16 in Fig. 2 or 2, 5, and 8 relative to the spanwise domain). The $k_{x}$ enstrophy spectra, in contrast, have their major contributions at somewhat lower wavenumbers, since the convective rolls are much longer than they are wide (but inclined along the phase of the wave).

By $t=70$, the dominant enstrophy has shifted to wavenumbers $k_{x} \sim 2$ to 20 and $k_{y} \sim 2$ to 30 (in a variance-content form, the peaks would occur at a $k_{x}$ for which the slope in Fig. 2 is -1$)$. At later times $(t=75$ and 80$)$, the earlier peaks in the $k_{y}$ spectra corresponding to the streamwise convective rolls are no longer present, as the vortex loops have interacted and driven the flow toward a more chaotic and isotropic structure.

Also shown in each panel of Fig. 2 are lines having spectral slopes of $+1 / 3$ and -5 , corresponding to the slopes one would expect for enstrophy in the inertial and viscous ranges of turbulence given energy spectral slopes of $-5 / 3$ and -7 , the -7 being from the Heisenberg model (Heisenberg, 1948). In the viscous range, the agreement with the -5 slope is reasonably good, except very near the Nyquist scales where the slope is steeper. In the inertial range, the spectra are flat in- 

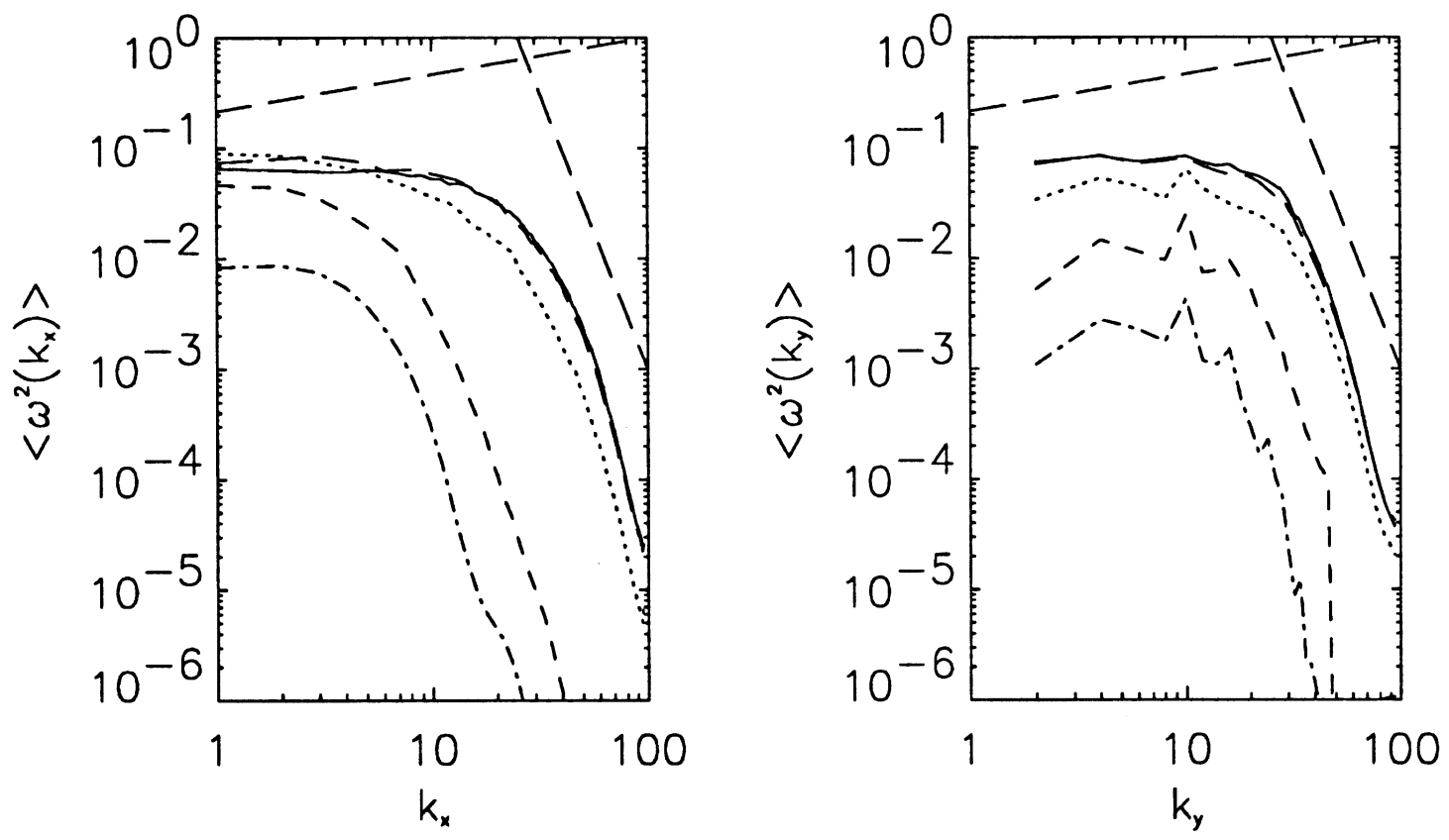

Fig. 2. Streamwise (left) and spanwise (right) wavenumber spectra at times of $t=60$ (dash-dotted), 65 (short-dashed), 70 (dotted), 75 (long-dashed), and 80 (solid) showing the approach toward isotropy at intermediate and smaller scales of motion. Also shown in each panel are lines having slopes of $+1 / 3$ and -5 .

stead of positively sloped; an exception is at lower wavenumbers and early times in the spanwise spectra where the spectra are positively sloped. The limited extent and slope of the apparent inertial range are likely consequences of a turbulent Reynolds number of 10 or less based on either integral scale or turbulence arguments (Fritts et al., 1996a). Nevertheless, the clear change in slope in the spectra at $k \sim 30$ is suggestive of the transition from an inertial range to a dissipative range.

\section{Summary and Conclusions}

We have presented an analysis of the vorticity dynamics accompanying initial convective instability, secondary dynamical instability, and the subsequent transition to and cascade within turbulence due to a breaking internal gravity wave simulated with a three-dimensional, high-resolution numerical model. The gravity wave was excited in a lower model domain and propagated into a higher-resolution upper domain having a streamwise wind shear designed to confine wave instability to the domain interior. Open boundary conditions permitting outward propagation of wave energy were used at the lower and upper boundaries of the lower and upper domains, respectively, and periodic boundary conditions were used at the lateral boundaries. Model parameters were chosen to be representative of wave propagation and instability in the middle atmosphere. Our simulation thus describes a common means by which turbulence arises in geophysical flows.

Initial convective instability within the wave field proceeds through the development of streamwise counter-rotating vortices arising due to baroclinic vorticity generation within the convectively unstable phase of the wave. These streamwise vortices evolve immediately above the large spanwise vor- ticity due to the superposition of wave and mean velocity shears. Strain due to these streamwise vortices contributes in several ways to the subsequent evolution of the spanwise vorticity layer. Stretching of the spanwise vorticity in regions of spanwise-divergent flow below adjacent streamwise vortices leads to thinning and intensification of this vorticity locally. The streamwise vortices also contribute to the generation of vertical vorticity through tilting the edges of each evolving spanwise vortex sheet.

Secondary dynamical (Kelvin-Helmholtz) instabilities develop on each of the intensified spanwise vortex sheets, serving to concentrate the spanwise vorticity into vortex tubes. At the edges of each spanwise vortex sheet, tilting of vertical vorticity into the streamwise direction by the developing vortex tubes acts to connect each tube with the two counterrotating streamwise vortices accounting for intensification of that vortex sheet. The net result of the initial convective and secondary dynamical instabilities is a series of intertwined vortex loops having increasingly complex geometries and interactions with time.

We have also examined the primary and secondary vortex interactions and perturbations accounting for the transition to and the enstrophy cascade within a turbulent flow following initial convective and secondary dynamical instability. Vortex interactions judged to be of primary importance in this context include the stretching of vortex structures (sheets and tubes) by nearly orthogonal neighboring vortex structures, the roll-up of vortex sheets into tubes, and mode-zero and mode-two twist waves which are excited on the vortex tubes. Other interactions also identified in our simulation, but judged to be of lesser importance in the evolution of the vorticity field, include reconnection, mode-one twist waves, and vortex pairing. 
A typical sequence of events begins with orthogonal vortex structures stretching each other. If one of the structures is a vortex sheet, the intensification due to the stretching eventually causes it to roll up into one or more vortex tubes. However, the vortex sheet is finite in extent, and so the resulting vortex tubes have variations in their structure along their axes. These axial variations excite twist waves along the tubes, particularly mode-zero and mode-two waves, and these twist waves have such large amplitudes that they fragment, unravel, or burst the tubes on which they propagate. Twist waves can also be excited by interactions of vortex tubes with their neighbors, leading to the same disastrous consequences for the tubes. In this way, the flow evolves from sheets to tubes to tube fragments, each on smaller scales. The process is somewhat self-sustaining as we find that the tube fragments themselves stretch sheets which in turn roll up into new tubes; such processes occur until late in the evolution when the flow is primarily viscously diffusing. Mutual stretching events termed " $x$-patterns" were found to be relatively stable structures and to persist for long times.

Secondary vortex interactions are evident throughout the flow evolution, but appear to play a less significant role in the cascade of enstrophy toward smaller scales of motion. Reconnection events occur at many sites, although on some occasions apparent reconnection events proved to be nothing more than the intensification of separate portions of a vortex tube which already shared common vorticity fieldlines. Mode-one twist waves are occasionally excited, usually by the effect of an orthogonal neighboring vortex. These waves were found to be relatively stable and steady, probably because of the steady forcing from the neighboring tube. Finally, vortex pairing events were observed at several sites in our numerical results and were found to contribute to vortex perturbations leading to their fragmentation and bursting. But the pairing events themselves appeared to play a lesser role than other processes in a less viscous environment.

Perhaps the most significant result of this study is the identification of vortex processes which we believe to be responsible for dynamics within a broad class of turbulent flows. Turbulence has, of course, been studied in many contexts, and the details of the transition to turbulence vary according to which flow instabilities contribute to the transition. However, a common feature shared by many, if not all, turbulent flows is the formation of either vortex tubes or vortex loops, termed "horseshoe" or "hairpin" vortices (Rogers and Moin, 1987; Robinson, 1991; Sandham and Kleiser, 1992; Gerz et al., 1994; Metais et al., 1995). Similar structures also occur in the general 3D KH instability (Palmer et al., 1996) and in many other flows of sufficiently high Reynolds numbers. The dynamics of the vortex loops that arise in our current simulation, then, are likely representative of similar dynamics of vortex loops in many flows. For example, the twist waves we observe on vortex loops are caused by axial variations of the vortex loops (see Arendt et al., 1997, for a full discussion) and by interactions with neighbors. As such variations inevitably occur on finite-length vortex tubes or loops, twist waves and the consequent fragmentation and/or unraveling of the vortex loops should be common in turbulent flows. In fact, double helix unraveling of vortex tubes has been observed by Cadot et al. (1995) in laboratory stud- ies of turbulent shear flow. In our flows, we find that twist waves are ubiquitous, and the fragmentation and unraveling of vortex loops that they cause is the predominant fate of the loops; few, if any, vortex loops decay by viscous diffusion.

Acknowledgments. This research was supported by the Norwegian Defence Research Establishment, the National Science Foundation under grants ATM-9419151 and ATM-9618004, and the Air Force Office of Scientific Research under grants F49620-96-10300 and F49620-98-C-0029. We are grateful to James Garten for assistance in preparing several of the figures used in the paper. Volumetric visualization was performed using the Viz software package developed by P. $\varnothing$. Hvidsten of NDRE.

\section{References}

Acarlar, M. S. and C. R. Smith, A study of hairpin vortices in a laminar boundary layer. Part II: Hairpin vortices generated by fluid injection, $J$. Fluid Mech., 175, 43-83, 1987.

Andreassen, Ø., C. E. Wasberg, D. C. Fritts, and J. R. Isler, Gravity wave breaking in two and three dimensions, 1. Model description and comparison of two-dimensional evolutions, J. Geophys. Res., 99, 8095-8108, 1994a.

Andreassen, Ø., I. Lie, and C. E. Wasberg, The spectral viscosity method applied to simulation of waves in a stratified atmosphere, J. Comp. Phys., 110, 257-273, 1994b.

Andreassen, Ø., P. Ø. Hvidsten, D. C. Fritts, and S. Arendt, Vorticity dynamics in a breaking gravity wave 1 : Initial instability evolution, $J$. Fluid Mech., 367, 27-46, 1998.

Arendt, S., D. C. Fritts, and Ø. Andreassen, The initial value problem for Kelvin vortex waves, J. Fluid Mech., 344, 181-212, 1997.

Betchov, R., On the fine structure of turbulent flows, J. Fluid Mech., 3, 205-216, 1957.

Boratav, O. N., R. B. Pelz, and N. J. Zabusky, Reconnection in orthogonally interacting vortex tubes: Direct numerical simulations and quantifications, Phys. Fluids A, 4, 581-605, 1992.

Cadot, O., S. Douady, and Y. Couder, Characterization of the low-pressure filaments in a three-dimensional turbulent shear flow, Phys. Fluids A, 7, 630-646, 1995.

Canuto, C., M. Y. Hussaini, A. Quarteroni, and T. A. Zang, Spectral Methods in Fluid Dynamics, 567 pp., Springer, 1988.

Caulfield, C. P. and W. R. Peltier, Three dimensionalization of the stratified mixing layer, Phys. Fluids A, 6, 3803-3805, 1994.

Davis, P. A. and W. R. Peltier, Some characteristics of the Kelvin-Helmholtz and resonant overreflection modes of shear instability and of their interaction through vortex pairing, J. Atmos. Sci., 36, 2394-2412, 1979.

Drazin, P. G., The stability of a shear layer in an unbounded heterogeneous inviscid fluid, J. Fluid Mech., 4, 214-224, 1958.

Dunkerton, T. J., Inertio-gravity waves in the stratosphere, J. Atmos. Sci., 41, 3396-3404, 1984.

Dunkerton, T. J., Shear instability of internal inertia-gravity waves, J. Atmos. Sci., 54, 1628-1641, 1997.

Erlebacher, G. and S. Sarkar, Statistical analysis of the rate of strain tensor in compressible homogeneous turbulence, Phys. Fluids A, 5, 3240-3254, 1993.

Fritts, D. C., Shear excitation of atmospheric gravity waves. Part II: Nonlinear radiation from a free shear layer, J. Atmos. Sci., 41, 524-537, 1984.

Fritts, D. C. and P. K. Rastogi, Convective and dynamical instabilities due to gravity wave motions in the lower and middle atmosphere: Theory and observations, Radio Sci., 20, 1247-1277, 1985.

Fritts, D. C. and L. Yuan, Stability analysis of inertio-gravity wave structure in the middle atmosphere, J. Atmos. Sci., 46, 1738-1745, 1989.

Fritts, D. C., J. R. Isler, and Ø. Andreassen, Gravity wave breaking in two and three dimensions, 2 . Three dimensional evolution and instability structure, J. Geophys. Res., 99, 8109-8123, 1994.

Fritts, D. C., J. F. Garten, and Ø. Andreassen, Wave breaking and transition to turbulence in stratified shear flows, J. Atmos. Sci., 53, 1057-1085, $1996 a$.

Fritts, D. C., T. L. Palmer, Ø. Andreassen, and I. Lie, Evolution and breakdown of Kelvin-Helmholtz billows in stratified compressible flows, I: Comparison of two- and three-dimensional flows, J. Atmos. Sci., 53, 3173-3191, 1996b.

Fritts, D. C., J. R. Isler, J. H. Hecht, R. L. Walterscheid, and Ø. Andreassen, Wave breaking signatures in sodium densities and $\mathrm{OH}$ airglow, Part II: 
Simulation of wave and instability structures, J. Geophys. Res., 102, 6669-6684, 1997.

Fritts, D. C., S. Arendt, and $\varnothing$. Andreassen, Vorticity dynamics in a breaking internal gravity wave, 2. Vortex interactions and transition to turbulence, J. Fluid Mech., 367, 47-65, 1998.

Gerz, T., Coherent structures in stratified turbulent shear flows deduced from direct simulations, in Turbulence and Coherent Structures, edited by O. Metais and M. Lesieur, Kluwar, Dordrecht, the Netherlands, 1991.

Gerz, T., J. Howell, and L. Mahrt, Vortex structures and microfronts, Phys. Fluids A, 6, 1242-1251, 1994.

Heisenberg, W., Zur statistischen theorie der turbulenz, Z. Physik, 124, 628657,1948

Herring, J. R. and R. M. Kerr, Development of enstrophy and spectra in numerical turbulence, Phys. Fluids A, 5, 2792-2798, 1993.

Hill, R. J., D. E. Gibson-Wilde, J. A. Werne, and D. C. Fritts, Turbulenceinduced fluctuations in ionization and application to PMSE, Earth Planets Space, 51, this issue, 499-513, 1999.

Hodges, R. R., Jr., Generation of turbulence in the upper atmosphere by internal gravity waves, J. Geophys. Res., 72, 3455-3458, 1967.

Hopfinger, E. J., F. K. Browand, and Y. Gagne, Turbulence and waves in a rotating tank, J. Fluid Mech., 125, 505-534, 1982.

Isler, J. R., D. C. Fritts, and Ø. Andreassen, Gravity wave breaking in two and three dimensions 3. Vortex breakdown and transition to isotropy, $J$. Geophys. Res., 99, 8125-8137, 1994.

Jeong, J. and F. Hussain, On the identification of a vortex, J. Fluid Mech., 285, 69-94, 1995.

Jimenez, J., Hyperviscous vortices, J. Fluid Mech., 279, 169-176, 1994.

Jimenez, J., A. A. Wray, P. G. Saffman, and R. S. Rogallo, The structure of intense vorticity in isotropic turbulence, J. Fluid Mech., 255, 65-90, 1993.

Kelvin, Lord, Vibrations of a columnar vortex, Phil. Mag., 10, 155-168, 1880.

Kida, S. and M. Takaoka, Vortex reconnection, Ann. Rev. Fluid Mech., 26, 169-189, 1994.

Klaassen, G. P. and W. R. Peltier, The onset of turbulence in finite-amplitude Kelvin-Helmholtz billows, J. Fluid Mech., 155, 1-35, 1985.

Kline, S. J., W. C. Reynolds, F. A. Schraub, and P. W. Runstadler, The structure of turbulent boundary layers, J. Fluid Mech., 30, 741-773, 1967.

Koop, C. G. and F. K. Browand, Instability and turbulence in stratified fluid with shear, J. Fluid Mech., 93, 135-159, 1979.

Landahl, M. T. and E. Mollo-Christensen, Turbulence and Random Processes in Fluid Mechanics, Cambridge Univ. Press., 1992.

LeLong, M.-P. and T. J. Dunkerton, Inertia-gravity wave breaking in three dimensions, 1. Convectively stable waves, J. Atmos. Sci., 55, 2473-2488, 1998a.

LeLong, M.-P. and T. J. Dunkerton, Inertia-gravity wave breaking in three dimensions, 2. Convectively unstable waves, J. Atmos. Sci., 55, 24892501,1998 b.

Lundgren, T. S., Strained spiral vortex model for turbulent fine structure, Phys. Fluids, 25, 2193-2203, 1982

Maxworthy, T., E. J. Hopfinger, and L. G. Redekopp, Wave motions on vortex cores, J. Fluid Mech., 151, 141-165, 1985.

Melander, M. V. and F. Hussain, Core dynamics on a vortex column, Fluid Dyn. Res., 13, 1-37, 1994.

Melander, M. V. and F. Hussain, Polarized vorticity dynamics on a vortex column, Phys. Fluid A, 5, 1992-2003, 1995.
Metais, O., C. Flores, S. Yanase, J. Riley, and M. Lesieur, Rotating freeshear flows. Part 2. Numerical simulations, J. Fluid Mech., 293, 47-80, 1995.

Metcalfe, R. W., S. A. Orsag, M. E. Brachet, S. Menon, and J. J. Riley, Secondary instability of a temporally growing mixing layer, J. Fluid Mech., 184, 207-243, 1987.

Palmer, T. L., D. C. Fritts, Ø. Andreassen, and I. Lie, Three-dimensional evolution of Kelvin-Helmholtz billows in stratified compressible flow, Geophys. Res. Lett., 21, 2287-2290, 1994.

Palmer, T. L., D. C. Fritts, and Ø. Andreassen, Secondary convective and dynamical instabilities of Kelvin-Helmholtz billows in three-dimensional stratified compressible flow, J. Fluid Mech., submitted, 1996.

Patnaik, P. C., F. S. Sherman, and G. M. Corcos, A numerical simulation of Kelvin-Helmholtz waves of finite amplitude, J. Fluid Mech., 73, 215-240, 1976.

Pumir, A. and E. Siggia, Collapsing solutions to the 3-D Euler equations, Phys. Fluids A, 2, 220-241, 1990.

Robinson, S. K., Coherent motions in the turbulent boundary layer, Аnnu. Rev. Fluid Mech., 23, 601-639, 1991.

Rogers, M. M. and P. Moin, The structure of the vorticity field in homogeneous turbulent flows, J. Fluid Mech., 176, 33-66, 1987.

Saffman, P. G., Vortex Dynamics, 311 pp., Cambridge., 1992.

Sandham, N. D. and L. Kleiser, The late stages of transition to turbulence in channel flow, J. Fluid Mech., 245, 319-348, 1992.

Schoppa, W., F. Hussain, and R. W. Metcalfe, A new mechanism of small scale transition in a plane mixing layer: core dynamics of spanwise vortices, J. Fluid Mech., 298, 23-80, 1995.

Scinocca, J. F., The mixing of mass and momentum by Kelvin-Helmholtz billows, J. Atmos. Sci., 52, 2509-2530, 1995.

Scotti, R. S. and G. M. Corcos, An experiment on the stability of small disturbances in a stratified free shear layer, J. Fluid Mech., 52, 499-528, 1972 .

She, Z. S., E. Jackson, and S. A. Orszag, Intermittent vortex structures in homogeneous isotropic turbulence, Nature, 344, 226-228, 1990.

Shelley, M. J., D. I. Meiron, and S. A. Orszag, Dynamical aspects of vortex reconnection of perturbed anti-parallel vortex tubes, J. Fluid Mech., 246, 613-652, 1993.

Sonmor, L. J. and G. P. Klaassen, Toward a unified theory of gravity wave stability, J. Atmos. Sci., 54, 2655-2680, 1997.

Thorpe, S. A., Laboratory observations of secondary structures in KelvinHelmholtz billows and consequences for ocean mixing, Geophys. Astrophys. Fluid Dyn., 34, 175-199, 1985.

Vincent, A. and M. Meneguzzi, The spatial structure and statistical properties of homogeneous turbulence, J. Fluid Mech., 225, 1-20, 1991.

Vincent, A. and M. Meneguzzi, The dynamics of vorticity tubes in homogeneous turbulence, J. Fluid Mech., 258, 245-254, 1994.

Winters, K. B. and E. A. D'Asaro, 3D wave breaking near a critical level, J. Fluid Mech., 272, 255-284, 1994.

Winters, K. B. and J. Riley, Instability of internal waves near a critical level, Dyn. of Atmos. and Oceans, 16, 249-278, 1992.

Yuan, L. and D. C. Fritts, Influence of a mean shear on the dynamical instability of an inertio-gravity wave, J. Atmos. Sci., 46, 2562-2568, 1989.

D. C. Fritts (e-mail: dave@co-ra.com), S. Arendt, and Ø. Andreassen 